\title{
Crystal structure and fluorescence properties of the iSpinach aptamer in complex with DFHBI
}

\author{
PABLO FERNANDEZ-MILLAN, ALEXIS AUTOUR, ERIC ENNIFAR, ERIC WESTHOF, and MICHAEL RYCKELYNCK \\ Université de Strasbourg, CNRS, Architecture et Réactivité de I'ARN, UPR 9002, F-67000 Strasbourg, France
}

\begin{abstract}
Fluorogenic RNA aptamers are short nucleic acids able to specifically interact with small molecules and strongly enhance their fluorescence upon complex formation. Among the different systems recently introduced, Spinach, an aptamer forming a fluorescent complex with the 3,5-difluoro-4-hydroxybenzylidene imidazolinone (DFHBI), is one of the most promising. Using random mutagenesis and ultrahigh-throughput screening, we recently developed iSpinach, an improved version of the aptamer, endowed with an increased folding efficiency and thermal stability. iSpinach is a shorter version of Spinach, comprising five mutations for which the exact role has not yet been deciphered. In this work, we cocrystallized a reengineered version of iSpinach in complex with the DFHBI and solved the X-ray structure of the complex at $2 \AA$ resolution. Only a few mutations were required to optimize iSpinach production and crystallization, underlying the good folding capacity of the molecule. The measured fluorescence half-lives in the crystal were $60 \%$ higher than in solution. Comparisons with structures previously reported for Spinach sheds some light on the possible function of the different beneficial mutations carried by iSpinach.
\end{abstract}

Keywords: fluorogenic RNA aptamer; DFHBI; crystal structure; fluorescence

\section{INTRODUCTION}

The discovery of naturally fluorescent proteins had a profound impact on biology by making it possible to track proteins in the cell, but also monitoring gene expression as well as devising new innovative fluorogenic sensors (Enterina et al. 2015; Mishin et al. 2015). Even though no naturally fluorescent RNA has been discovered yet, several artificial fluorogenic RNAs have been developed. These RNAs are short nucleic acids able to specifically interact with small molecules that become fluorescent upon complex formation. This capacity of an RNA molecule to light up a fluorogenic dye was first demonstrated with the aptamer selected to specifically interact with malachite green (Grate and Wilson 1999), which was later found to be able to efficiently activate the fluorescence of triphenylmethane dyes (Babendure et al. 2003). Since then, a variety of alternative fluorogenic dye/ RNA aptamer couples have been developed (Dolgosheina and Unrau 2016; Ouellet 2016; Pauff et al. 2017). Similarly to fluorescent proteins, fluorescent RNAs have a wide application spectrum (e.g., live-cell gene expression imaging [Guet et al. 2015; Zhang et al. 2015], biosensing of proteins [Song et al. 2013], nucleic acids [Sato et al. 2015; Aw et al. 2016; Huang et al. 2017; Ong et al. 2017], or even metabolites [Nakayama et al. 2012; Paige et al. 2012; Kellenberger et al.

Corresponding author: m.ryckelynck@unistra.fr

Article is online at http://www.rnajournal.org/cgi/doi/10.1261/rna. 063008.117. Freely available online through the RNA Open Access option.
2013, 2015a,b; Sharma et al. 2014; You et al. 2015; Bose et al. 2016; Hallberg et al. 2016; Su et al. 2016; Wang et al. 2016]), and one can anticipate their significant impact on RNA biology.

Among the variety of fluorogenic aptamers currently known, Spinach is the best characterized and the most widely used. This RNA was originally identified by in vitro selection of aptamers for their capacity to interact with the 3,5difluoro-4-hydroxybenzylidene imidazolinone (DFHBI), a GFP-mimicking dye that is nontoxic, bio-orthogonal, and cell permeable (Paige et al. 2011). Further engineering of the original aptamer allowed the development of Spinach 2, a molecule with increased folding properties and thermal stability (Strack et al. 2013). Structural characterization of both DFHBI-binding aptamers revealed that they are organized around a three-tier G-quadruplex core forming the bottom layer of the DFHBI-binding pocket that accommodates the dye in planar conformation (Huang et al. 2014; Warner et al. 2014). The remainder of the DFHBI-binding pocket consists of a top layer made of a base-triple while the side of the dye establishes interactions with nucleobases. Despite the tight accommodation of the dye and the improved properties of Spinach 2, we recently found that the folding properties of the aptamer were still suboptimal (Autour et al. 2016). To further optimize Spinach RNA, we

(C) 2017 Fernandez-Millan et al. This article, published in RNA, is available under a Creative Commons License (Attribution-NonCommercial 4.0 International), as described at http://creativecommons.org/licenses/by-nc/4.0/. 
generated mutant libraries and screened them for brighter and better folding mutants using our recently introduced microfluidic-assisted in vitro compartmentalization pipeline (Ryckelynck et al. 2015). During this process we used selection pressures (i.e., warming, complete absence of $\mathrm{K}^{+}$and use of $\mathrm{Na}^{+}$instead) aimed at challenging RNA structural stability, especially that of the G-quadruplex core (Autour et al. 2016). Sequence analysis of the most fluorescent mutants allowed us to identify several beneficial mutations, of which the five displaying the highest effect were combined into the same aptamer. Further engineering the molecule led to an improved Spinach molecule (iSpinach), a DFHBIbinding aptamer forming a fluorescent complex that is characterized by a high thermal stability, a reduced salt-sensitivity, and an increased brightness. Interestingly, none of the selected mutations occurred in the DFHBI-binding pocket, suggesting that they may primarily have an effect on aptamer folding.

In the present work, we determined the crystal structure of iSpinach in complex with the DFHBI to gain insight into the role of the different mutations introduced in the aptamer. Our data indicate that, as anticipated, the mutations act at the structural level by compacting and stabilizing the structure of the aptamer. More specifically, we found that the replacement of an AoA base pair interacting via a single hydrogen bond and connecting the G-quadruplex to the helix distal from the DFHBI-binding site by a GoG base pair forming a Watson-Crick/Hoogsteen interaction allows a better coordination of potassium by the G-quadruplex through the formation of a water-mediated network.

\section{RESULTS AND DISCUSSION}

\section{Molecular engineering and crystallogenesis}

The original iSpinach aptamer was first reengineered to optimize its production and to promote intermolecular interactions during the crystallization process. First, the double mutation $\mathrm{C} 2 \mathrm{G} / \mathrm{C} 5 \mathrm{G}$ was introduced at the $5^{\prime}$ end of the sequence of all the constructs to increase the in vitro transcription reaction yield (Supplemental Fig. S1) by providing an optimal internal transcription sequence to the enzyme; the consequence was only the inversion of the Watson-Crick base pair $\mathrm{C}=\mathrm{G}$ by $\mathrm{G}=\mathrm{C}$ in the basal stem (Fig. 1). Second,

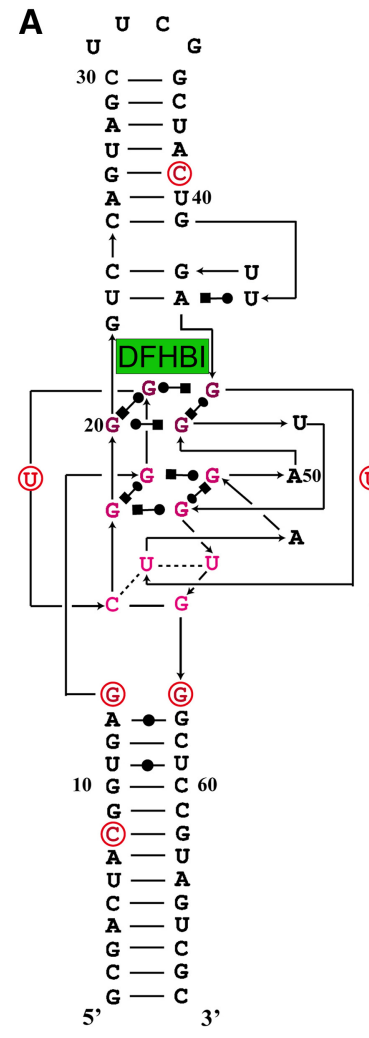

iSpinach -WT

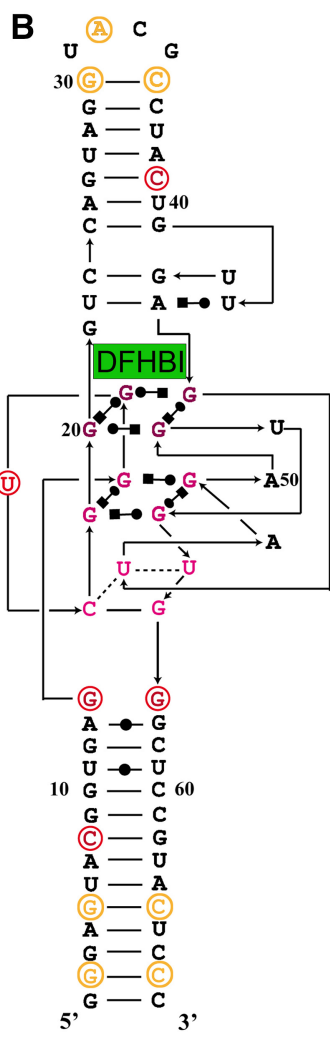

iSpinach-D5-30G-32A

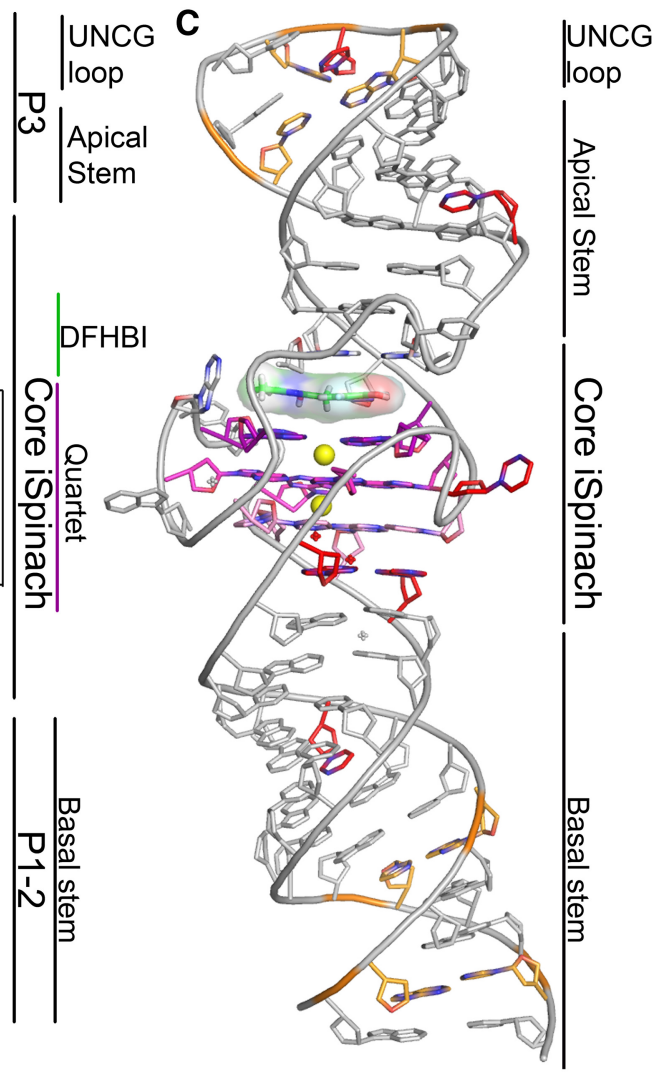

iSpinach-D5-30G-32A

FIGURE 1. Structural organization of iSpinach and its crystallized mutant. (A) Secondary structure of iSpinach. (B) Secondary structure of iSpinachD5-G30-A32. On both models, the beneficial mutations combined to obtain iSpinach are shown and circled in red, while those introduced to increase the transcription rate of the molecule and promote its crystallization are shown in orange. $(C)$ Crystal structure of iSpinach-D5-G30-A32. The residues are shown using the same color code used on secondary structure models. Potassium ions are shown as yellow spheres. 
the second position of the UUCG tetraloop capping the apical hairpin was varied (UNCG motif) in order to promote intermolecular contacts in the crystal and favor the crystallization success. Finally, it is reported that the two base pairs proximal to the UNCG may affect its folding and stability (Molinaro and Tinoco 1995; Davis and Znosko 2007). Therefore, we assayed both the original base pair $\mathrm{C}=\mathrm{G}$ and a $\mathrm{G}=\mathrm{C}$ mutant. All tested constructs conserved intact fluorogenic properties and several of them cocrystallized in the presence of DFHBI (Supplemental Tables S1, S2), especially iSpinach-D5-G30-A32, which yielded crystals diffracting at a resolution of 2-2.4 $\AA$, a resolution exceeding that obtained in previous studies. Remarkably, iSpinach readily crystallized without the assistance of a protein as reported previously (Huang et al. 2014), underlying the improved folding capacity of the aptamer. This makes iSpinach the first DFHBIbinding aptamer crystallized in its native state with minor sequence changes and without the need for sequence insertion (Huang et al. 2014) or cleavage (Warner et al. 2014).

As a quality control, we first verified the fluorescence of the crystallized iSpinach-D5-G30-A32/DFHBI complex by fishing one crystal from each crystallization condition and, after washing, imaging the crystal by epi-fluorescence microscopy (Fig. 2A). We then evaluated the photostability of crystal and soluble complexes by placing them within a capillary under continuous illumination at DFHBI excitation wavelength (i.e., $475 \mathrm{~nm}$ ) while monitoring fluorescence decay (Fig. 2B). To avoid artifactual fluorescence recovery of the soluble complex through the diffusion of fluorescent complexes from a nonilluminated area of the capillary, the solution of RNA/ DFHBI complex was encapsulated into $100 \mathrm{pL}$ water-in-oil droplets using a microfluidic droplet generator (Anna et al. 2003) and the resulting emulsion was loaded into the capillary (Fig. 2A). As expected from the difference in local concentration, the fluorescence intensity of the crystallized complex largely exceeded that of the soluble complex. Nevertheless, normalizing the fluorescence to that of the starting exposure time revealed that both types of complexes displayed similar fluorescence half-life, with the crystallized form having a fluorescence half-life only twice higher than the soluble one (Fig. 2B). Moreover, we observed that crystals melted and cracked during the experiment, suggesting conformational changes consistent with a DFHBI photo-inactivation model in which the dye isomerizes upon illumination and is rapidly ejected from the aptamer (Han et al. 2013). Altogether, these results suggest that the crystallized form of the complex is representative of its native state in solution.

\section{Crystal structure of the complex}

The iSpinach-D5-G30-A32 molecule folds into an $80.5 \AA$ long double-stranded hairpin, containing in its center a Gquadruplex surrounded by two sets of coaxially stacked helical stems. The hairpin is capped by a tetraloop composed of UACG, from U31 to G34, that adopts a conventional fold for

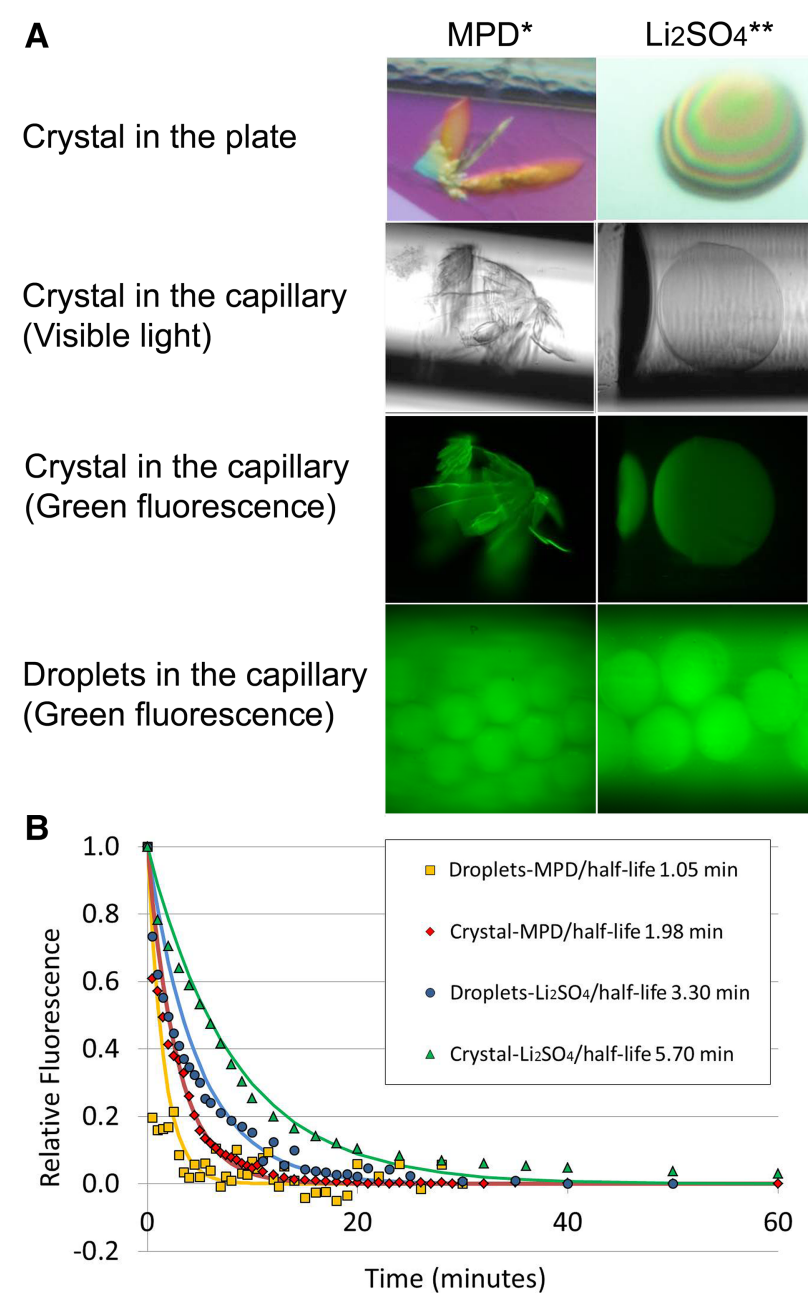

FIGURE 2. Fluorescence properties of the iSpinach-D5-G30-A32/ DFHBI crystallized complex. (A) Micrographs of crystals and droplets. Micrographs of the crystals were taken while they were in the crystallization plate or loaded into the capillary and observed under visible light or in epi-fluorescence mode. The conditions in which the crystal was grown or the droplets prepared (MPD or $\mathrm{Li}_{2} \mathrm{SO}_{4}$ ) are indicated under the corresponding column. $\left({ }^{*}\right)$ MPD crystallization conditions were $32 \% \mathrm{MPD}, 40 \mathrm{mM} \mathrm{NaCl}, 40 \mathrm{mM}$ sodium cacodylate $\mathrm{pH} 7.0$ and 12 $\mathrm{mM}$ spermine. (**) $\mathrm{Li}_{2} \mathrm{SO}_{4}$ crystallization conditions were $1.9 \mathrm{M}$ $\mathrm{Li}_{2} \mathrm{SO}_{4}, 50 \mathrm{mM}$ MES pH 5.6, $5 \mathrm{mM} \mathrm{MgCl}_{2}$. (B) Fluorescence decay monitoring. The fluorescence of the crystals or the droplets was monitored along the time. The values were relativized to that of the first measurement point, and an exponential decay was fit to the data to compute the fluorescence half-life (values given in the legend of the plot).

UNCG loops where G34 interacts with the ribose edge of U31 with an extra H-bond to the A32 ribose (Ennifar et al. 2000). Moreover, C33 (N4) forms an H-bond to an anionic A32 oxygen atom. Therefore, as initially expected, modulating the sequence of this tetraloop promoted crystal packing since the exposed A32 forms a base-pair kissing contact with a neighboring iSpinach molecule (Supplemental Fig. S2D). Besides A32, U43 and A48 are also exposed to the solvent and involved in the crystal packing. Both U43 and A48 from two different neighboring molecules and related by 
crystallographic symmetry are interacting together by a double set of interactions: first through stacking interaction and second by H-bonds between hydroxyl A48( $\left.\mathrm{O}^{\prime}\right)$ and $\mathrm{U} 43$ (O4). At the same time, U43 and A48 have contacts with a third molecule: U43(O2) interacts with G19(N2), A48(N1) with $\mathrm{G} 55\left(\mathrm{O}_{2}^{\prime}\right)$, and $\mathrm{A} 48(\mathrm{~N} 1)$ with $\mathrm{G} 55(\mathrm{~N} 3)$ by H-bonds (Supplemental Fig. S2E). Finally, the first base pair of the basal stem, G1=C69, interacts through stacking interaction with the homologous base pair of another RNA molecule (Supplemental Fig. S2F).

The functional core of iSpinach-D5-G30-A32 is located at the center of the molecule and defines the DFHBI binding site. The core is composed of two structural groups: an ensemble of three quadruplex layers and one layer of triple base pairs, with the DFHBI being sandwiched in between these two structural groups.

The quadruplexes are composed of two regular G-quartet layers and a third mixed layer (Fig. 3). In the double G-quartet layer, the top layer (layer 1 ) is defined by the interaction of G20 and G16 with an anti glycosyl conformation, while G46 and G51 are in the syn glycosyl conformation. The second quartet (layer 2) is composed of G49 in the anti glycosyl conformation with G15, G19, and G53 in the syn glycosyl conformation. The third layer, below layer 2, is a mixed sequence tetrad (layer 3) in which C18 and G55 bind as a Watson-

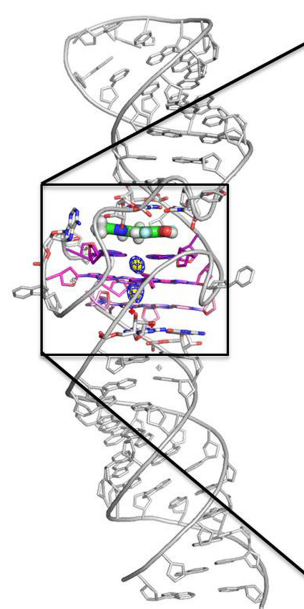

layer1

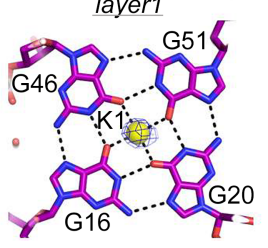

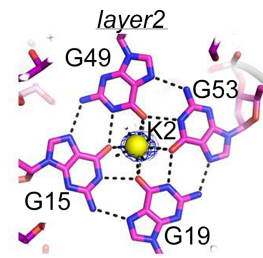
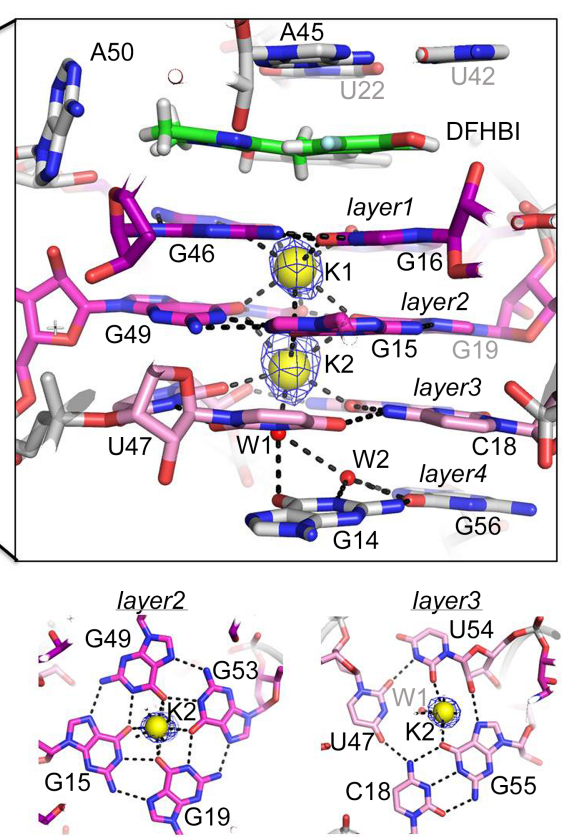

Crick pair with, in addition, U47 pairing with $\mathrm{C} 18$ in the anti glycosyl conformation and U54 in the syn glycosyl conformation binding G55. Using anomalous data collected at $1.8 \AA$ wavelength $\left(f^{\prime} \sim 1.5 \overline{\mathrm{e}}\right)$, the nature and position of potassium ions were unambiguously confirmed by peaks at $6-$ $\sigma$ level in anomalous difference maps. A first potassium ion (K1) with octahedron coordination is found in between the first two layers, in agreement with the previous structural studies (Huang et al. 2014; Warner et al. 2014). Moreover, we identified a second potassium ion (K2) located between "layers 2 and 3." This ion also has an octahedron coordination through the participation of a water molecule (W1) fixed by the bases of "layer 3" with the participation of G14 and G56 (Fig. 3), two mutations specific of iSpinach (see below).

The second structural motif forming the DFHBI-binding site is a triple base pair interaction in planar orientation (Lid layer) where U22 and A45 interact through canonical Watson-Crick pair while U42 and A45 form a WatsonCrick/Hoogsteen cis base pair (Fig. 3). This triple forms the upper part of the DFHBI-binding pocket into which the DFHBI intercalates in a planar conformation and makes extensive aromatic stacking interactions with the first G-quartet layer. In addition to stacking interaction with layer 1 of the Gquartet, the phenol group of the DFHBI makes an interaction involving its hydroxyl and the ribose hydroxyl of G16. Moreover, the imidazole ring of the dye makes direct contacts with A50 and G21. The high resolution of our structure allows the unambiguous fit of the active cis isomer of the DFHBI in the density map (Supplemental Fig. S3), further confirming that the complex is in its native form.

Finally, the center core of the molecule is flanked by two helical regions composed of Watson-Crick interactions involving nucleotides $1-14$ and 56-69 in the basal stem and nucleotides $24-30$ in 35-41 in the apical stem, the latter being capped by a tetraloop.

\section{Structural comparative analysis of iSpinach and Spinach aptamers}

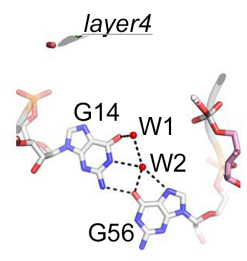

FIGURE 3. iSpinach crystallographic structure. The crystal structure of iSpinach-D5-G30-A32 is shown in light gray (top left) together with a zoom on the core region of the molecule involved in DFHBI binding. The ligand DFHBI is colored in green. The quartet motif is in magenta from dark magenta in layer 1 , magenta layer 2 , and light magenta layer 3 . Potassium ions are represented by yellow spheres and the anomalous differential electronic density maps ( $\mathrm{Fo}-\mathrm{Fc}$ at $6.0 \sigma$ level) are shown as a blue mesh around the spheres. The interactions are represented by black dashed lines. The waters are represented by red dots. All the zoomed layers are shown perpendicular to the helical axis as well.
The overall structure of iSpinach/DFHBI complex is very similar to that reported for Spinach 1 and 2 aptamers, suggesting that the beneficial mutations contained in iSpinach may have subtle yet important effects. To shed light on the contribution of these mutations, we compared the structure obtained in the present study with those previously reported with different RNA constructs (Huang et al. 2014; Warner et al. 2014). To this end, 
we selected from each study the structure of the aptamer in complex with DFHBI having the highest resolution (i.e., PDB code 4KZD.pdb [Huang et al. 2014] and 4TS0.pdb [Warner et al. 2014]). Hereafter, these structures will be called, respectively, Spinach-P and Spinach-FdA for ease of reading (Supplemental Fig. S4).

The iSpinach core region spans from the G14-G56 base pair to the triple base pairs that define the lid layer of the DFHBI-binding pocket (Fig. 1). The superposition of the three structures (Fig. 4) indicates that they are very similar in their core region as highlighted by the very low RMSD (Supplemental Table S4). Consistently, the position and the relative orientation of the DFHBI inside the core of the RNA aptamer do not show differences between the three structures (Supplemental Fig. S5). Yet, interesting differences were observed. First, U17 in iSpinach (originally A24 in Spinach-P and A27 in Spinach-FdA) is exposed to the solvent (Fig. 4, bottom left), making a clear-cut interpretation of the effect of interchanging a purine for a pyrimidine challenging. Nevertheless, the distance between the OP1 phosphate of $\mathrm{U} 17$ and the $\mathrm{O} 2^{\prime}$ of $\mathrm{U} 42$ ribose is $3.6 \AA$ (U42 is involved in Hoogsteen triple interaction in the Lid layer of DHFBI), a distance significantly shorter than in Spinach-FdA $(4.3 \AA)$ and in Spinach-P $(4.5 \AA)$ and compatible with the formation of an electrostatic contact. Therefore, even though additional experiments will be required to conclude on the exact contribution of U17, our data suggest that this residue could act by increasing the folding stability of the DFHBI-binding pocket.
This mutation was also found to be the only one tolerated in the G-quadruplex region and its introduction in the original Spinach aptamer was also found to increase the fluorescence of the complex (Ketterer et al. 2015). Moreover, Broccoli, another DFHBI-binding aptamer (Filonov et al. 2014) proposed to share the same core structure as Spinach (Ageely et al. 2016), also possesses a $U$ at the same position, further supporting the key role of this residue. Second, of the three structures, the present one is the only one in which both potassium ions of the G-quartet core are seen with the proper coordination state. Indeed, in Spinach-P only the first potassium ion (K1) was observed between layers 1 and 2, while a water molecule occupies the $\mathrm{K} 2$ position. In addition, even though both K1 and K2 ions are seen in Spinach-FdA structure, the coordination of $\mathrm{K} 2$ is only seven. On the contrary, in the present structure a water molecule (W1) coordinating $\mathrm{K} 2$ is clearly observed on the density map (Fig. 3). W1 is held in place by an interaction network involving a second water molecule (W2) and the residues G14 and G56. Interestingly, in Spinach these residues are two adenines (A14 and A56) interacting by a single $\mathrm{H}$-bond between N1 and N6 in both structures (Fig. 4, top right). Conversely, in iSpinach, there are bifurcated H-bonds between G14 (N1) and G56 (O6) with G56 (N2), while W2 mediates four Hbonds between this base pair and layer 3 (Fig. 3). Regarding the impact of these mutations, 4KZD and 4KZE PDB structures (Huang et al. 2014) provide important information. The comparison of the DFHBI-bound Spinach-P

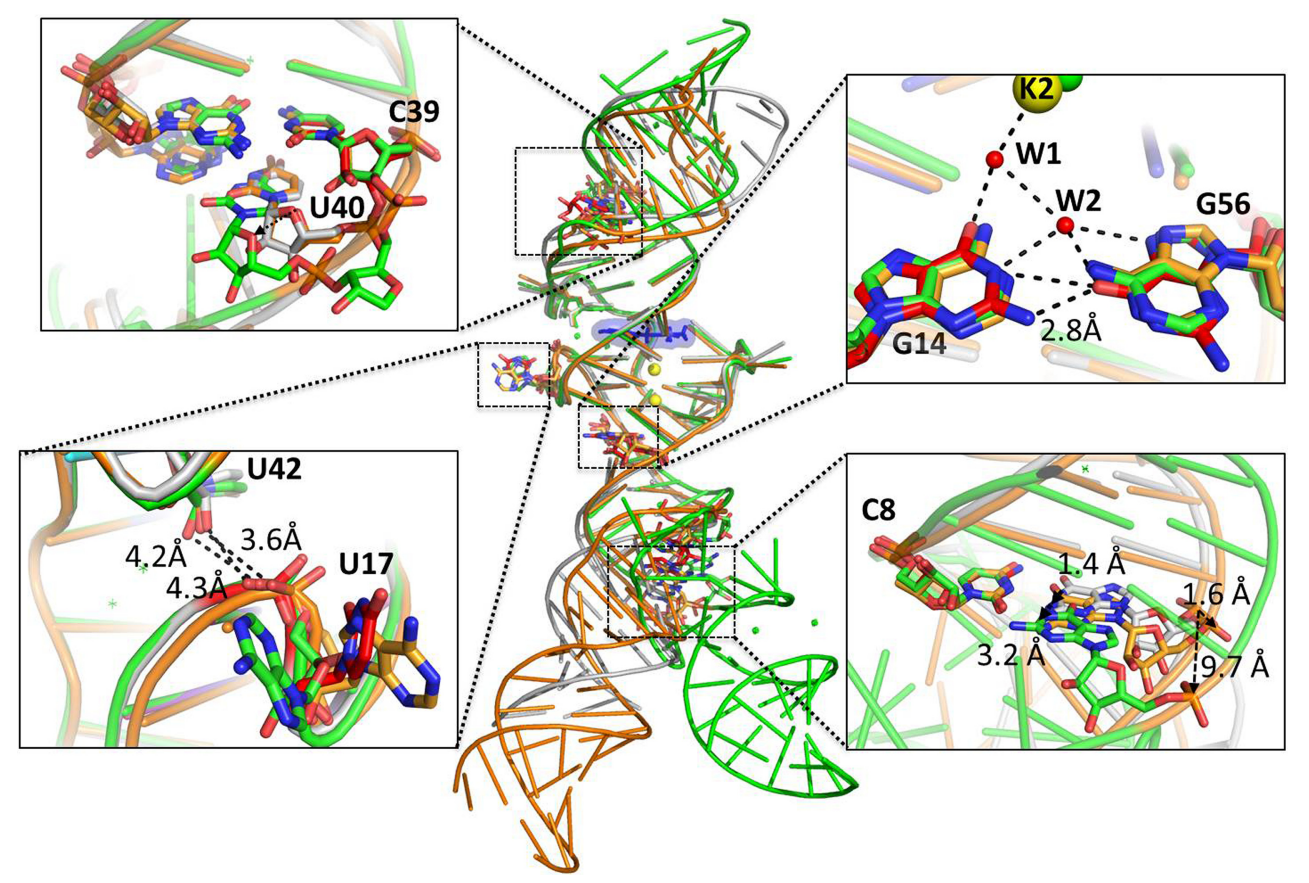

FIGURE 4. Superposition of different Spinach/DFHBI complex crystal structures. The structure obtained in the present study is shown in gray and is superimposed to that of Spinach-FdA (shown in green) and that of Spinach-P (shown in orange). The regions containing the beneficial mutations are zoomed-in and the mutated residues are shown in red, whereas the wild-type ones are shown according to the color code used before. Potassium ions are represented by yellow spheres and water molecules by red dots. 
(4KZD) with the DFHBI-free Spinach-P (4KZE) shows the substitution of U54 by A48 in layer 3. It is possible that a movement consequent to DFHBI binding promotes the loss of K2 coordination. Therefore, we suggest that GoG has a positive impact during this transition by holding the $\mathrm{K} 2$ in position via the water $\mathrm{W} 1$ and helping to stabilize the G-quartet core. This observation is consistent with the fact that, in our original screen, the two G's were always found together (Autour et al. 2016). This G pair was also recently found to increase the fluorescence of Broccoli, but at the expense of an increased dependency on magnesium (Ageely et al. 2016). The possibility that $\mathrm{W} 2$ is an ion (magnesium or sodium) was taken into account during the refinement process; however, this option was rejected after an exhaustive analysis because a correct coordination geometry was absent (Supplemental Fig. S6; Zheng et al. 2009, 2017).

Extending the superimposition of the three structures beyond the core significantly increased the RMSD values as a consequence of the variations in the stems of the RNAs. Indeed, the basal stems have different orientations with respect to the G-quadruplex domains, which are especially accentuated in the Spinach-FdA structure (in green on Fig. 4). Furthermore, the apical stem is even more challenging to compare because the designs of the different aptamers are completely different. Indeed, while in iSpinach the apical stem is closed by a UNCG tetraloop, in Spinach-P the loop is replaced by an RNA element bound to an antibody, and the Spinach-FdA structure results from the assembly of two single strands (Supplemental Fig. S4). Nevertheless, local superimpositions of the three structures allowed us to further decipher the contribution of the two left mutations (C39 and C8). As expected for the conversion of a weak GoU into a more stable $\mathrm{G}=\mathrm{C}$ pair, no structural difference was observed at the level of the C39 mutation (Fig. 4, top left). This confirms our initial suggestion (Autour et al. 2016) that the evolutionary process selected the mutation U39C only to increase the stability of the helix without modifying its structure. Since iSpinach and Spinach-P shared the same sequence from G28-C39 to the first layer of G-quartet, both molecules superposed well over this region. Conversely, the equivalent region of Spinach-FdA (G36-C57/U58/A35-U59) displays a bulged $\mathrm{U}$ residue that slightly disturbs the continuity of the helix. Finally, the $\mathrm{U}$ to $\mathrm{C}$ substitution at position 8 produces a better interaction with the $\mathrm{G}$ of the complementary strand. The $\mathrm{GoU}$ pair promotes the displacement of the complementary base by $1.4 \AA$ and $3.2 \AA$ (measured from N1 of the base) and a displacement of $1.6 \AA$ and $9.7 \AA$ in Spinach-P and Spinach-FdA structures, respectively (Fig. 4, bottom right). Overall, the major groove is wider in the Spinach-FdA structure due to the presence of a 2-nt bulge (Supplemental Fig. S7).

In conclusion, the cocrystal structure of iSpinach in complex with the DFHBI helps in the understanding of the contribution of the different mutations combined in the new aptamer. Indeed, whereas C8 and C39 were found to have the anticipated stabilization action by changing GoU pairs into more stable G=C's, the effect of U17, G14, and G56 were less obvious a priori. Nevertheless, our model now allows us to propose that these mutations could, at least in part, act by forming a new electrostatic interaction (U17) as well as ensuring a tighter coordination of the second potassium ion of the G-quartet via a water molecule (G14 and G56). The net effect of these different mutations is to compact and stabilize the structure of the aptamer, especially that of the Gquadruplex core and of the DFHBI binding pocket, making the iSpinach/DFHBI complex far less salt sensitive and more thermostable than the parental molecule as previously observed (Autour et al. 2016). This improved folding capacity is also supported by the fact that we could obtain diffracting crystals with minimal modification of the sequence. Indeed, only two base-pair inversions were introduced in the basal stem to optimize RNA synthesis, and a single point mutation was introduced in the apical loop to promote crystal-packing interactions. This clearly contrasts with previous work in which the RNA was formed from two half-molecules annealed together (Warner et al. 2014), or where the apical loop was substituted by a Fab-binding domain (Huang et al. 2014). This capacity of iSpinach to crystallize in its near-native state suggests a high structural homogeneity inside the population of molecules underlining the good folding capacity of iSpinach. Nevertheless, despite this improved folding capacity, iSpinach still shares the complex structural organization of the Spinach family (i.e., a mixed-base quadruplex core surrounded by two flanking A-form helices), characterized by a complex connectivity. Whereas building the dye-binding pocket around a base quadruple is common to several fluorogenic RNA aptamers (Trachman et al. 2017b), the use of more conventional G-quadruplex folding with short propeller arms may enable the connectivity of these molecules to be simplified by using, for instance, a simple GNRA-derived motif as recently described for Mango RNA (Trachman et al. 2017a). Therefore, future work may lead to the isolation of fluorogenic aptamers with folding properties even surpassing that of iSpinach. Finally, the present structure will also be of great interest for future experiments aimed at engineering the aptamer, for instance to convert iSpinach into an allosteric fluorogenic RNA.

\section{MATERIALS AND METHODS}

\section{iSpinach RNA synthesis}

The different primers used are summarized in Supplemental Table S1 and were obtained from Integrated DNA Technologies. For each construct, 0.1 pmol of template was introduced in $100 \mu \mathrm{L}$ of a PCR mixture containing $0.2 \mathrm{mM}$ of each $\mathrm{dNTP}, 0.2 \mu \mathrm{M}$ of each primer (forward and reverse), $0.1 \mathrm{U} / \mu \mathrm{L}$ of DreamTaq (ThermoFisher), and the corresponding buffer at the recommended working concentration. The mixture was then thermocycled starting with an initial denaturation step of $30 \mathrm{sec}$ at $95^{\circ} \mathrm{C}$ followed by 25 cycles of $5 \mathrm{sec}$ at $95^{\circ} \mathrm{C}$ and $30 \mathrm{sec}$ at $60^{\circ} \mathrm{C}$. Upon thermocycling, the $100 \mu \mathrm{L}$ of PCR mixture were introduced in $1 \mathrm{~mL}$ of in vitro transcription mixture 
containing $2 \mathrm{mM}$ of each NTP, $25 \mathrm{mM} \mathrm{MgCl}_{2}, 40 \mathrm{mM}$ Tris- $\mathrm{HCl} \mathrm{pH}$ 8.0, $5 \mathrm{mM}$ DTT, $1 \mathrm{mM}$ Spermidine, $70 \mu \mathrm{g} / \mathrm{mL}$ T7 RNA polymerase, and $5 \mu \mathrm{g} / \mathrm{mL}$ inorganic pyrophosphatase (Roche). After $6 \mathrm{~h}$ of incubation at $37^{\circ} \mathrm{C}, 10$ units of Baseline-Zero DNase (Epicentre) and the corresponding buffer were added to the mixture and a second incubation of $1 \mathrm{~h}$ at $37^{\circ} \mathrm{C}$ was performed. RNA was recovered by phenol extraction followed by an ethanol precipitation in the presence of $300 \mathrm{mM}$ sodium acetate $\mathrm{pH} 5.5$ (Sigma-Aldrich) and 3 volumes of ice cold $100 \%$ ethanol overnight at $-20^{\circ} \mathrm{C}$. After centrifugation and a wash in $70 \%$ ethanol, the pellets were dissolved in denaturing buffer $(0.05 \%$ bromophenol blue, $0.05 \%$ xylene cyanol, $20 \%$ glycerol, TBE $1 \times, 8 \mathrm{M}$ urea) and the solution loaded on a $12 \%$ denaturing $8 \mathrm{M}$ urea acrylamide/bisacrylamide gel. The piece of gel containing the RNA was identified by UV shadowing, sliced from the gel, and transferred into a BioTrap device (GE Healthcare) filled with TBE buffer. RNA was eluted for $3 \mathrm{~h}$ at $200 \mathrm{~V}$ and $4^{\circ} \mathrm{C}$ prior to being precipitated in the presence of $300 \mathrm{mM}$ sodium acetate $\mathrm{pH} 5.5$ (SigmaAldrich) and 3 volumes of ice cold $100 \%$ ethanol overnight at $-20^{\circ}$ C. RNA was then recovered by centrifugation, the pellet washed with $70 \%$ ethanol prior to being resuspended in deionized DEPC treatedwater (Roth). RNA was finally quantified by OD measurement at $260 \mathrm{~nm}$ using a NanoDrop device.

As a quality control, the RNA was analyzed on an $8 \%$ denaturing 8 $\mathrm{M}$ urea acrylamide/bisacrylamide gel. Moreover, $2 \mu \mathrm{M}$ of RNA were renaturated in water by 2 min of warming at $85^{\circ} \mathrm{C}$ followed by a cooling of $5 \mathrm{~min}$ at $25^{\circ} \mathrm{C}$ using a thermocycler before adding a volume of $2 \times$ incubation buffer $\left(80 \mathrm{mM}\right.$ Tris- $\mathrm{HCl} \mathrm{pH} \mathrm{7.5,2} \mathrm{mM} \mathrm{MgCl}_{2}$, and $200 \mathrm{mM} \mathrm{KCl}$ ) and DFHBI (Lucerna Technologies) to a final concentration of $1.2 \mu \mathrm{M}$. Finally, the fluorescence was measured in a realtime Thermocycler (Mx3005P, Agilent) on the FAM channel (ex: $492 \mathrm{~nm} / \mathrm{em}: 516 \mathrm{~nm}$ ) of the device.

\section{RNA/DFHBI cocrystallization}

A solution of $210 \mu \mathrm{M}$ of RNA was renaturated as described above and mixed 1:1.2 with DFHBI in a solution containing $40 \mathrm{mM}$ Tris- $\mathrm{HCl} \mathrm{pH} \mathrm{7.5,} 1 \mathrm{mM} \mathrm{MgCl}$, and $100 \mathrm{mM} \mathrm{KCl}$.

In crystallization conditions consisting of $\mathrm{Li}_{2} \mathrm{SO}_{4}(1.9-1.6 \mathrm{M}) \mathrm{MES}$ $\mathrm{pH} 5.4 \mathrm{MgCl}_{2}(15-5 \mathrm{mM})$ at $20^{\circ} \mathrm{C}$, both iSpinach-D5 and iSpinachD5-G30-A32 crystallized with the same shape: lentils/ball shape. A second set of crystals was obtained in MPD (28\%-34\%), sodium spermine (10-14 mM), $12 \mathrm{mM}$ Tris- $\mathrm{HCl} \mathrm{pH} 7.4$, and $\mathrm{NaCl}(40-$ $90 \mathrm{mM}$ ) at $20^{\circ} \mathrm{C}$ and $30^{\circ} \mathrm{C}$, but with two different shapes: For iSpinach-D5, only needles were observed, while iSpinach-D5-G30 A32 had a laminar shape crystal. In this crystallization condition, small elliptic crystals were also found with iSpinach-D5-G30-G32.

A solution of $2.0 \mathrm{M} \mathrm{Li}_{2} \mathrm{SO}_{4}, 50 \mathrm{mM}$ MES pH 5.4, $5 \mathrm{mM} \mathrm{MgCl}_{2}$ and $20 \%$ glycerol was used as cryo-protector for the first crystallization conditions; while a solution of $36 \% \mathrm{MPD}, 12 \mathrm{mM}$ sodium spermine, $40 \mathrm{mM}$ sodium cacodylate $\mathrm{HCl} \mathrm{pH} \mathrm{7.0,60} \mathrm{mM} \mathrm{NaCl}$ and $10 \%$ glycerol was used for the second one. Samples in both conditions were frozen in liquid ethane and stored in liquid nitrogen.

\section{Data collection and processing}

Data were collected at the X06DA beamline at the SLS using a PILATUS 2M detector. The wavelength was set to 1.8 A. Several data sets were collected for "lentils/ball shape" crystals for iSpinach-D5 and iSpinach-D5-G30-A32 constructs as well as for iSpinach-D5-G30-A32 with "laminar" crystal shape. Whereas most of the crystals had very poor (or no) diffraction (Supplemental Table S2), the "laminar" crystal obtained with iSpinach-D5-G30-A32 allowed the structure of the complex to be solved. All data sets collected from the "laminar" crystal were processed and scaled independently with XDSGUI (Kabsch 2010). All of them showed the same space group and cell dimension, therefore all were merged together in one data set for the refinement process with XDSGUI software. For phase determination, one of the Spinach structures solved previously (PDB code: 4Q9R.pdb, Huang et al. 2014) was used as a search model for molecular replacement using PHASER (PHENIX) (Adams et al. 2010). The final structure was obtained after alternative cycles of refinement with PHENIX and manual refinement with COOT (Supplemental Table S3; Emsley and Cowtan 2004). The final refined model was deposited on the PDB under the reference 5OB3.

\section{iSpinach fluorescence comparison between solution and crystal}

The crystals obtained with iSpinach-D5-G30-A32 construct in both crystallographic conditions $\left(\mathrm{Li}_{2} \mathrm{SO}_{4}\right.$ and MPD) were washed 3-4 times in respective crystallographic condition from the reservoir (Supplemental Table S2) in order to remove RNA molecules left in the solution. A single crystal was loaded into a Crystal Clear Special Glass 10 capillary (Hampton Research, $0.4 \mathrm{~mm}$ inner diameter).

For complexes kept in solution, $2 \mu \mathrm{M}$ of iSpinach-D5-G30-A32 was mixed with $2.4 \mu \mathrm{M}$ DFHBI in $40 \mathrm{mM}$ Tris- $\mathrm{HCl} \mathrm{pH} \mathrm{7.5,} 1$ $\mathrm{mM} \mathrm{MgCl}_{2}$ and $100 \mathrm{mM} \mathrm{KCl}$ and, afterwards, mixed at $50 \%$ with their respective crystallogenesis buffer (Supplemental Table S2). The mixture was then emulsified into $100 \mathrm{pL}$ water-in-oil droplets using Novec $7500(3 \mathrm{M})$ fluorinated oil supplemented with $2 \%$ of fluorosurfactant and using a microfluidic device as described previously (Autour et al. 2016). The collected emulsions were then loaded into the same capillary as those used with crystals. Fluorescence intensity decay was monitored by placing the loaded capillaries onto the platform of an epi-fluorescence microscope (TiE, Nikon) and exposing it to a constant illumination at $475 \mathrm{~nm}$ at the maximum intensity of the light source (Lumencor). An Orca Flash 4 camera using a proper filter set was used to collect the light emitted at 490-530 nm and the fluorescence was quantified using custommade analysis software written in MatLab.

\section{SUPPLEMENTAL MATERIAL}

Supplemental material is available for this article.

\section{ACKNOWLEDGMENTS}

We thank Redmond Smyth for his help in manuscript proofreading and Guillaume Bec for his assistance in using the Mosquito liquid handling robot. This work has been published under the framework of the LABEXs: ANR-10-LABX-0036_NETRNA and ANR-11LABX-0057_MITOC ROSS, and benefits from funding by the state-managed French National Research Agency as part of the Investments for the Future program. We also received the financial support of the Agence Nationale de la Recherche (project BacNet: ANR-10-BINF-02-01), the Université de Strasbourg, and the Centre National de la Recherche Scientifique.

Received July 16, 2017; accepted September 5, 2017. 


\section{REFERENCES}

Adams PD, Afonine PV, Bunkóczi G, Chen VB, Davis IW, Echols N, Headd JJ, Hung LW, Kapral GJ, Grosse-Kunstleve RW, et al. 2010. PHENIX: a comprehensive Python-based system for macromolecular structure solution. Acta Crystallogr D Biol Crystallogr 66: 213-221.

Ageely EA, Kartje ZJ, Rohilla KJ, Barkau CL, Gagnon KT. 2016. Quadruplex-flanking stem structures modulate the stability and metal ion preferences of RNA mimics of GFP. ACS Chem Biol 11: 2398-2406.

Anna SL, Bontoux N, Stone HA. 2003. Formation of dispersions using "flow focusing" in microchannels. Appl Phys Lett 82: 364-366.

Autour A, Westhof E, Ryckelynck M. 2016. iSpinach: a fluorogenic RNA aptamer optimized for in vitro applications. Nucleic Acids Res 44: 2491-2500.

Aw SS, Tang MX, Teo YN, Cohen SM. 2016. A conformation-induced fluorescence method for microRNA detection. Nucleic Acids Res 44: e92.

Babendure JR, Adams SR, Tsien RY. 2003. Aptamers switch on fluorescence of triphenylmethane dyes. J Am Chem Soc 125: 14716-14717.

Bose D, Su Y, Marcus A, Raulet DH, Hammond MC. 2016. An RNAbased fluorescent biosensor for high-throughput analysis of the cGAS-cGAMP-STING pathway. Cell Chem Biol 23: 1539-1549.

Davis AR, Znosko BM. 2007. Thermodynamic characterization of single mismatches found in naturally occurring RNA. Biochemistry 46: 13425-13436.

Dolgosheina EV, Unrau PJ. 2016. Fluorophore-binding RNA aptamers and their applications. Wiley Interdiscip Rev RNA 7: 843-851.

Emsley P, Cowtan K. 2004. Coot: model-building tools for molecular graphics. Acta Crystallogr D Biol Crystallogr 60: 2126-2132.

Ennifar E, Nikulin A, Tishchenko S, Serganov A, Nevskaya N, Garber M, Ehresmann B, Ehresmann C, Nikonov S, Dumas P. 2000. The crystal structure of UUCG tetraloop. J Mol Biol 304: 35-42.

Enterina JR, Wu L, Campbell RE. 2015. Emerging fluorescent protein technologies. Curr Opin Chem Biol 27: 10-17.

Filonov GS, Moon JD, Svensen N, Jaffrey SR. 2014. Broccoli: rapid selection of an RNA mimic of green fluorescent protein by fluorescence-based selection and directed evolution. J Am Chem Soc 136: 16299-16308.

Grate D, Wilson C. 1999. Laser-mediated, site-specific inactivation of RNA transcripts. Proc Natl Acad Sci 96: 6131-6136.

Guet D, Burns LT, Maji S, Boulanger J, Hersen P, Wente SR, Salamero J, Dargemont C. 2015. Combining Spinach-tagged RNA and gene localization to image gene expression in live yeast. Nat Commun 6: 8882.

Hallberg ZF, Wang XC, Wright TA, Nan B, Ad O, Yeo J, Hammond MC. 2016. Hybrid promiscuous (Hypr) GGDEF enzymes produce cyclic AMP-GMP (3', 3'-cGAMP). Proc Natl Acad Sci 113: 1790-1795.

Han KY, Leslie BJ, Fei J, Zhang J, Ha T. 2013. Understanding the photophysics of the spinach-DFHBI RNA aptamer-fluorogen complex to improve live-cell RNA imaging. J Am Chem Soc 135: 19033-19038.

Huang H, Suslov NB, Li NS, Shelke SA, Evans ME, Koldobskaya Y, Rice PA, Piccirilli JA. 2014. A G-quadruplex-containing RNA activates fluorescence in a GFP-like fluorophore. Nat Chem Biol 10: 686-691.

Huang K, Doyle F, Wurz ZE, Tenenbaum SA, Hammond RK, Caplan JL, Meyers BC. 2017. FASTmiR: an RNA-based sensor for in vitro quantification and live-cell localization of small RNAs. Nucleic Acids Res 45: e130

Kabsch W. 2010. Integration, scaling, space-group assignment and postrefinement. Acta Crystallogr D Biol Crystallogr 66: 133-144.

Kellenberger CA, Wilson SC, Sales-Lee J, Hammond MC. 2013. RNAbased fluorescent biosensors for live cell imaging of second messengers cyclic di-GMP and cyclic AMP-GMP. J Am Chem Soc 135: 4906-4909.

Kellenberger CA, Chen C, Whiteley AT, Portnoy DA, Hammond MC. 2015a. RNA-based fluorescent biosensors for live cell imaging of second messenger cyclic di-AMP. J Am Chem Soc 137: 6432-6435.

Kellenberger CA, Wilson SC, Hickey SF, Gonzalez TL, Su Y, Hallberg ZF, Brewer TF, Iavarone AT, Carlson HK, Hsieh YF, et al. 2015b. GEMM-I riboswitches from Geobacter sense the bacterial second messenger cyclic AMP-GMP. Proc Natl Acad Sci 112: 5383-5388.
Ketterer S, Fuchs D, Weber W, Meier M. 2015. Systematic reconstruction of binding and stability landscapes of the fluorogenic aptamer spinach. Nucleic Acids Res 43: 9564-9572.

Mishin AS, Belousov VV, Solntsev KM, Lukyanov KA. 2015. Novel uses of fluorescent proteins. Curr Opin Chem Biol 27: 1-9.

Molinaro M, Tinoco I Jr. 1995. Use of ultra stable UNCG tetraloop hairpins to fold RNA structures: thermodynamic and spectroscopic applications. Nucleic Acids Res 23: 3056-3063.

Nakayama S, Luo Y, Zhou J, Dayie TK, Sintim HO. 2012. Nanomolar fluorescent detection of c-di-GMP using a modular aptamer strategy. Chem Commun (Camb) 48: 9059-9061.

Ong WQ, Citron YR, Sekine S, Huang B. 2017. Live cell imaging of endogenous mRNA using RNA-based fluorescence "Turn-On” probe. ACS Chem Biol 12: 200-205.

Ouellet J. 2016. RNA fluorescence with light-up aptamers. Front Chem 4: 29.

Paige JS, Wu KY, Jaffrey SR. 2011. RNA mimics of green fluorescent protein. Science 333: 642-646.

Paige JS, Nguyen-Duc T, Song W, Jaffrey SR. 2012. Fluorescence imaging of cellular metabolites with RNA. Science 335: 1194.

Pauff S, Withers JM, McKean IJ, Mackay SP, Burley GA. 2017. Synthetic biological approaches for RNA labelling and imaging: design principles and future opportunities. Curr Opin Biotechnol 48: 153-158.

Ryckelynck M, Baudrey S, Rick C, Marin A, Coldren F, Westhof E, Griffiths AD. 2015. Using droplet-based microfluidics to improve the catalytic properties of RNA under multiple-turnover conditions. RNA 21: 458-469.

Sato S, Watanabe M, Katsuda Y, Murata A, Wang DO, Uesugi M. 2015. Live-cell imaging of endogenous mRNAs with a small molecule. Angew Chem Int Ed Engl 54: 1855-1858.

Sharma S, Zaveri A, Visweswariah SS, Krishnan Y. 2014. A fluorescent nucleic acid nanodevice quantitatively images elevated cyclic adenosine monophosphate in membrane-bound compartments. Small 10: $4276-4280$.

Song W, Strack RL, Jaffrey SR. 2013. Imaging bacterial protein expression using genetically encoded RNA sensors. Nat Methods 10: 873-875.

Strack RL, Disney MD, Jaffrey SR. 2013. A superfolding Spinach2 reveals the dynamic nature of trinucleotide repeat-containing RNA. Nat Methods 10: 1219-1224.

Su Y, Hickey SF, Keyser SG, Hammond MC. 2016. In vitro and in vivo enzyme activity screening via RNA-based fluorescent biosensors for S-adenosyl-t-homocysteine (SAH). J Am Chem Soc 138: 7040-7047.

Trachman RJ III, Demeshkina NA, Lau MWL, Panchapakesan SSS, Jeng SCY, Unrau PJ, Ferré-D’Amaré AR. 2017a. Structural basis for high-affinity fluorophore binding and activation by RNA Mango. Nat Chem Biol 13: 807-813.

Trachman RJ III, Truong L, Ferré-D’Amaré AR. 2017b. Structural principles of fluorescent RNA aptamers. Trends Pharmacol Sci 38: 928-939.

Wang XC, Wilson SC, Hammond MC. 2016. Next-generation RNAbased fluorescent biosensors enable anaerobic detection of cyclic di-GMP. Nucleic Acids Res 44: e139.

Warner KD, Chen MC, Song W, Strack RL, Thorn A, Jaffrey SR, FerréD'Amaré AR. 2014. Structural basis for activity of highly efficient RNA mimics of green fluorescent protein. Nat Struct Mol Biol 21: 658-663.

You M, Litke JL, Jaffrey SR. 2015. Imaging metabolite dynamics in living cells using a Spinach-based riboswitch. Proc Natl Acad Sci 112: E2756-E2765.

Zhang J, Fei J, Leslie BJ, Han KY, Kuhlman TE, Ha T. 2015. Tandem spinach array for mRNA imaging in living bacterial cells. Sci Rep 5: 17295.

Zheng G, Lu XJ, Olson WK. 2009. Web 3DNA-a web server for the analysis, reconstruction, and visualization of three-dimensional nucleic-acid structures. Nucleic Acids Res 37: W240-W246.

Zheng H, Cooper DR, Porebski PJ, Shabalin IG, Handing KB, Minor W. 2017. CheckMyMetal: a macromolecular metal-binding validation tool. Acta Crystallogr D Struct Biol 73: 223-233. 

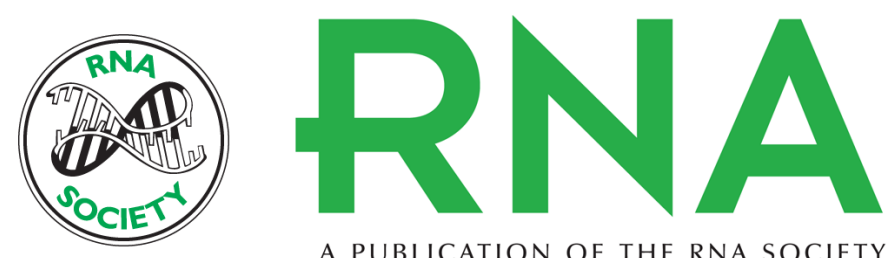

A PUBLICATION OF THE RNA SOCIETY

\section{Crystal structure and fluorescence properties of the iSpinach aptamer in complex with DFHBI}

Pablo Fernandez-Millan, Alexis Autour, Eric Ennifar, et al.

RNA 2017 23: 1788-1795 originally published online September 22, 2017

Access the most recent version at doi:10.1261/rna.063008.117

\section{Supplemental http://rnajournal.cshlp.org/content/suppl/2017/09/21/rna.063008.117.DC1 Material}

References This article cites 46 articles, 7 of which can be accessed free at: http://rnajournal.cshlp.org/content/23/12/1788.full.html\#ref-list-1

Open Access Freely available online through the RNA Open Access option.

Creative This article, published in $R N A$, is available under a Creative Commons License Commons (Attribution-NonCommercial 4.0 International), as described at License http://creativecommons.org/licenses/by-nc/4.0/.

Email Alerting Receive free email alerts when new articles cite this article - sign up in the box at the Service top right corner of the article or click here.

To subscribe to $R N A$ go to:

http://rnajournal.cshlp.org/subscriptions 